\title{
CONTROL-INFLUENCED LAYOUT OPTIMIZATION OF ARRAYS OF WAVE ENERGY CONVERTERS
}

\author{
Philip Balitsky \\ Giorgio Bacelli \\ John V. Ringwood \\ Centre for Ocean Energy Research, Centre for Ocean Energy Research, Centre for Ocean Energy Research, \\ National University of Ireland Maynooth National University of Ireland Maynooth National University of Ireland Maynooth \\ Co. Kildare, Ireland \\ Co. Kildare, Ireland \\ Co. Kildare, Ireland \\ Email: balitsky@eeng.nuim.ie \\ Email: giorgio.bacelli@eeng.nuim.ie \\ Email: john.ringwood@eeng.nuim.ie
}

\begin{abstract}
In this paper we compare the optimal configurations for an array of WECs given two control schemes, a real-time global control and a passive sea-state based tuning scheme. In a particular wave climate and array orientation with its axis normal to the prevailing wave direction, closely-spaced symmetrical arrays of 2, 3, 4, 5, and 6 cylinders of different radiative properties are simulated for varying inter-device separation distances. For each device and control type, we focus on the factors that influence the optimal layout, including number of devices, separating distance and angular spreading. The average annual power output is calculated for each optimal configuration.
\end{abstract}

\section{Nomenclature}

$\beta \quad$ incoming wave heading in WAMIT $\left({ }^{\circ}\left(^{\circ}\right)\right.$

$\delta \quad$ linearized viscous damping coefficient stopping criteria

$\dot{z} \quad$ heave velocity

$\mathbf{K}(t) \quad$ matrix of radiation impulse responses

M generalized mass matrix

$\mathrm{m}_{\infty} \quad$ value of added mass at infinite frequency

$\sigma_{p} \quad$ angular spreading at peak period $\left({ }^{\circ}\right)$

$\theta_{p} \quad$ wave direction at peak period $\left(^{\circ}\right)$

$\theta_{a v} \quad$ mean wave direction ${ }^{\circ}$

A the area of the body projected onto the plane orthogonal to the velocity $\left(\mathrm{m}^{2}\right)$

$B_{v} \quad$ linearized viscous damping coefficient $(\mathrm{kg} / \mathrm{s})$
$B_{\text {pto }} \quad$ passive tuning PTO damping coefficient $(\mathrm{kg} / \mathrm{s})$

$C_{d} \quad$ drag coefficient

$d \quad$ inter-device separation distance (m)

$D(f, \theta)$ directional spreading function

$G C \quad$ global control

$k \quad$ index of devices in the WEC array

$N \quad$ number of frequency components in control simulation

$P \quad$ power per wave crest length $(\mathrm{kW} / \mathrm{m})$

$P T \quad$ passive tuning to a single frequency

$r \quad$ device radius $(\mathrm{m})$

$s \quad$ non-dimensional directional spreading parameter

$S(f) \quad$ non-directional wave power spectrum $\left(\mathrm{m}^{2} / \mathrm{Hz}\right)$

PTO Power Take-Off

WEC Wave Energy Converter

\section{INTRODUCTION}

In order for wave energy to compete with other renewables at the utility scale, wave energy converters (WECs) will have to be deployed in arrays, as scaling up individual WECs to the several megawatts is economically impractical. Unlike offshore wind turbines, where the array layout is designed to minimize destructive wake effects, a WEC array offers the possibility to offset the destructive effects of shadowing through positive hydrodynamic interactions. Furthermore, unlike wind, which, although directional in nature, is similar in characteristics throughout the world, waves are shaped by physical processes that are 
site specific, for example the proximity to shore or the size of the generating basin. Therefore waves in different regions are dissimilar enough that each WEC array layout will have to be designed for a specific site.

Because of the cost of eventual wave farm infrastructure such as electrical cables and moorings, as well concerns for navigation and conflicts with other ocean users, wave energy farms will likely be designed to minimize inter-device spacing. Since interaction between devices is greatest at short separation distances, this presents an opportunity to utilize constructive interference to increase the power yield of an array. For many types of WECs radiated waves generated by their motion are significant enough to modify the power uptake of surrounding devices. An active control scheme can strategically modify the wave field to increase the power uptake of the whole array. However, because the phase condition that facilitates constructive interference between WECs is dependent upon the frequency and directional characteristics of the prevailing wave climate, the array layout needs to be designed based on both the wave climate and control scheme. The pioneering studies in control of arrays such as [1,2] were performed for regular waves only and in the frequency domain. Likewise, to date, the majority of the investigations into array layout, such as [3-6], considered only regular waves. More recently, a number of papers were published [7-12] that modelled the layout of arrays of WECs in more realistic conditions, with spectral seas and directional influences considered. However, these studies have not looked at optimal control, instead parametrizing the power take-off (PTO) as a linear damper. Following a slightly different approach, [13] investigated optimal control for an array in irregular seas by taking the maximum interaction factors for each wave component and averaging them. The first paper investigating various array layouts and separation distances for controlled devices was by the authors in [14], however, as is stated therein, the aim of that study was to compare control strategies for arrays rather than look at array layouts. Using wave data from the AMETS test site in Ireland, this article compares the optimal WEC array layouts created by implementing global array control (GC) [14] and sea-state based Passive Tuning (PT) [15] for 3 different heaving cylinders. PT was previously applied in array layout studies for regular waves in [4, 6, 13, 16, 17], where significant constructive interference was observed. We focus on short separation distances $(d \leq 40 r)$ and circular arrays for several reasons. Firstly, both beneficial and negative interaction decreases with increasing separation distance [14], secondly, given the possibility of sharing moorings and electrical infrastructure [12,18], closely-spaced configurations might offer an economic advantage regardless of hydrodynamics. Additionally, because they are less sensitive to changes in wave direction and frequency as well as device positioning, [18], circular arrays can offer a more predictable power output for a variable wave climate. We formulate the control problem in the time domain, which is then discretized and solved as a nonlinear program. While this is not necessary for the fundamental unconstrained case considered in this paper it provides the possibility to extend the comparison to the constrained case, considered in [19].

\section{WAVE CLIMATE AND SEA STATE MODELLING 2.1 Study area}

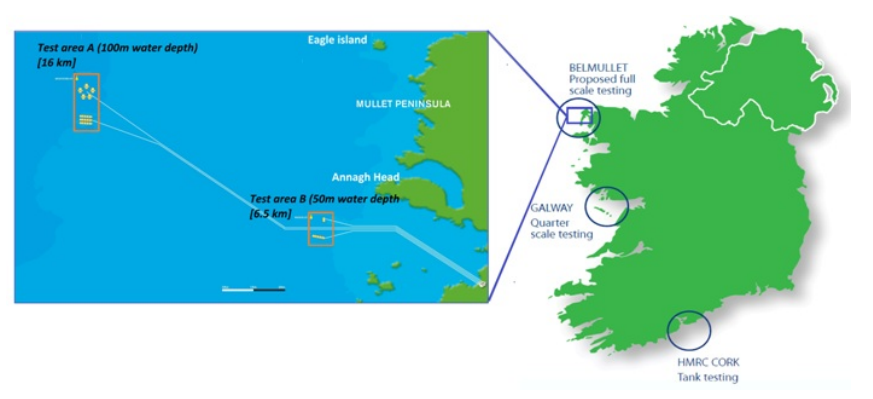

FIGURE 1. THE ATLANTIC MARINE ENERGY TEST SITE (AMETS) AND IRELAND'S WAVE ENERGY TESTING FACILITIES (Image courtesy of SEAI)

It is generally recognized that arrays of WECs will have to be designed for a specific deployment location [11]. As of 2013 there are no full scale array projects in the sea, nonetheless there are several sites in the planning stages. The Atlantic Marine Energy Test Site (AMETS) located off Annagh Head, west of Belmullet in County Mayo, Ireland, is one such site. Presently, the Westwave array demonstration project is in the planning stages at AMETS 1 .

\subsection{Measured wave data}

A Datawell Directional Waverider, a spherical, surfacefollowing measurement buoy, has been gathering data at the AMETS location since December 2009. This buoy is located at $54.2310^{\circ} \mathrm{N},-10.1460^{\circ} \mathrm{W}$ at the $50 \mathrm{~m}$ depth contour, designated as Berth B in Fig. 11 For the purposes of this study, a yearly time series covering the months of October 2010 through September 2011 has been chosen. The data, provided by the Marine Institute of Ireland, consist of a non-directional measured spectrum and summary directional statistics. The frequency range (in $\mathrm{rad} / \mathrm{s}$ ) is from 0.004 to 0.101 in increments of 0.7958 , and the data is collected at 3-minute intervals. A bi-variate scatter diagram of significant wave height versus the peak period or energy period is usually used to asses the performance of a wave energy device or array of devices at a specific site [12,20,21]. To create a scatter diagram for the AMETS site, we sort each 3 minute data set

\footnotetext{
${ }^{1}$ see WWw . westwave. ie for current project status
} 
according to $H_{m 0}$ and $T_{e}$. Excluding those sea states for which the probability of occurrence is less than .05\% accounts for $95 \%$ of the total energy. The resulting matrix is shown in Fig. 2. Since the Datawell buoy provides the zero-crossing period $T_{z}$ and not $T_{e}$, we have used a multiplying factor of 1.35 to obtain $T_{e}$ from $T_{z}$, following the procedure in Appendix A of [22]. Given the availability of spectral data for each time step, an average $S(f)$ was calculated from all the spectra in each scatter diagram bin.

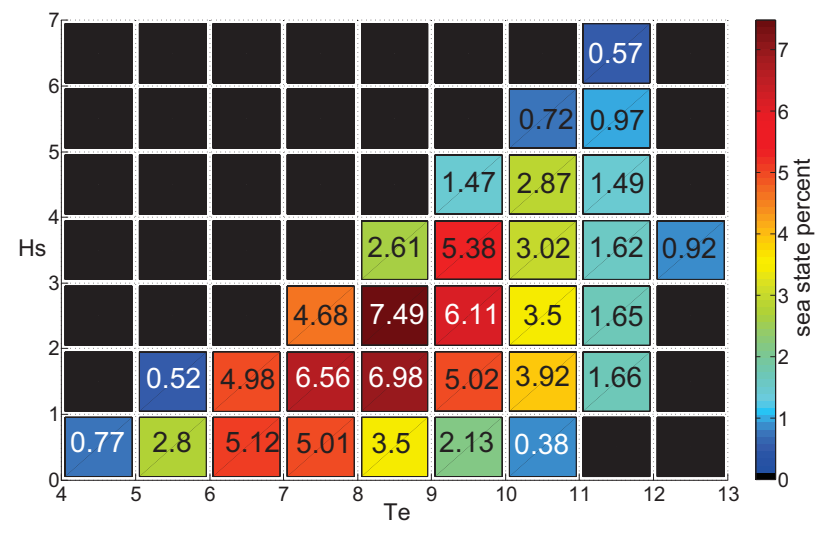

FIGURE 2. PERCENTAGE OCCURRENCE MATRIX AT THE AMETS TEST SITE OFF BELMULLET, CO. MAYO, IRELAND

\subsection{Summary statistics}

Relevant wave statistics for the AMETS site are shown in Tab. 1. From the statistic we can infer that the AMETS site is

TABLE 1. ATLANTIC MARINE ENERGY TEST SITE WAVE DATA SUMMARY Oct. 2010 - Sept. 2011

\begin{tabular}{|llllll|}
\hline$H_{m 0} a v(m)$ & 2.71 & $H_{m 0} \min$ & 0.49 & $H_{m 0} \max$ & 14.75 \\
$T_{p} a v(s)$ & 11.17 & $T_{p} \min$ & 4.75 & $T_{p} \max$ & 18.75 \\
$\operatorname{Pav}(k W / m)$ & 28.5 & $P \min$ & .36 & $P \max$ & 1459 \\
$\sigma_{p} a v\left(^{\circ}\right)$ & 30.44 & $\theta a v\left(^{\circ}\right)$ & 281.7 & & \\
\hline
\end{tabular}

very energetic, due to its exposure to the most powerful North Atlantic swells. This leads to a significant variability in the power available, indicating a probably need control the device motions. Indeed if we estimate the power per wave crest length for the study period, given by $P=0.49 H_{m 0}^{2} T_{e}$, which assumes deep water, $\rho=1025 \mathrm{~kg} / \mathrm{m}^{3}$ and $g=9.81 \mathrm{~m} / \mathrm{s}^{2}$, we obtain a maximum of $P=1459 \mathrm{~kW} / \mathrm{m}$, which is two orders of magnitude higher than the mean value of $28.5 \mathrm{~kW} / \mathrm{m}$, using $\rho=1025 \mathrm{~kg} / \mathrm{m}^{3}$ and assuming deep water. Given such high values of energy, it is recognized that a survival strategy will have to be utilized by the WECs, a factor that will be taken into consideration in setting the limits for the operational sea states in the results in section 5

\subsection{Directional parameters}

As demonstrated in many studies [3, 5], the power output of an array is sensitive to the direction of the incoming waves. Yet a large majority of current WEC designs and mooring systems do not have the capacity to change orientation to improve performance once the construction phase is over. By arranging the array layout so that an orientation which maximizes the power output follows $\theta_{a v}$, we can aim to capture the most energy possible in a multi-directional wave climate. Furthermore, at any given time a sea state will have angular spread that can be characterized as the standard deviation from the mean direction. The influence of directional spreading on array performance has been examined in a number of recent studies [7, 9], which showed a smoothing effect on array interactions. In this paper we take directional and spreading parameters into consideration by utilizing the well-known $\cos ^{2 s}$ formula, given in Eq. (1):

$$
D(f, \theta)=\frac{2^{2 s-1}}{\pi} \frac{\Gamma^{2}(s+1)}{\Gamma(2 s+1)} \cos ^{2 s}\left(\frac{\theta-\theta_{a v}}{2}\right)
$$

Here $\Gamma$ is the Gamma function, $\theta_{a v}$ is the mean direction of the incoming waves, and the spreading parameter $s$ models the directional width of the spectrum; since our wave data includes the spreading parameter $\sigma_{p}$, we can use a relation proposed in [23] that equates the two parameters. The resulting equation for $s$ is given by

$$
s=\frac{2}{\sigma_{p}^{2}}-1
$$

where $\sigma_{p}$ is given in radians. Since in this study we are tuning the devices to match the period of the dominant waves, we take the spread at the peak period, $\sigma_{p}$, to derive a value of 6.29 for $s$, comparable to that cited in [9] and [7]. The mean direction in Eq. (1) is set so that the array axis is oriented to match areas of constructive interference at close separation distances, as detailed in Sec. 5

\section{FORMULATION OF HYDRODYNAMIC MODEL}

The control systems are implemented on different array layouts and for several geometries of the WECs composing the arrays. The WECs are vertical cylinders of radius $r$, draft $h$ and the distance between their vertical axis is denoted with $d$.

\subsection{Equations of Motion}

For this work we assume linear wave theory and noncompressible irrotational flow. Therefore, the motion of the de- 
vice can be described, in the time domain, by Cummin's equation [24]:

$$
\mathbf{M}_{t} \ddot{z}(t)+\mathbf{B} \dot{z}(t)+\int_{t_{0}}^{t} \mathbf{K}(t-\tau) \dot{z}(\tau) d \tau+\mathbf{S} z(t)=f(t)
$$

where $z(t) \in \mathbb{R}^{m}$ is the vector of the vertical positions of the WECs, and $m$ the number of degrees of freedom of the system, which corresponds to the number of WECs composing the array, because the motion of each device is assumed to be restricted to heave only. $\mathbf{M}_{t}=\mathbf{M}+\mathrm{m}_{\infty}$ where $\mathbf{M} \in \mathbb{R}^{m \times m}$ is the generalised mass matrix and $\mathrm{m}_{\infty} \in \mathbb{R}^{m \times m}$ is the asymptotic values of the added mass at infinite frequency; $\mathbf{B} \in \mathbb{R}^{m \times m}$ is the viscous damping term; $\mathbf{S} \in \mathbb{R}^{m \times m}$ is the hydrodynamic stiffness and $\mathbf{K}(t) \in \mathbb{R}^{m \times m}$ is the matrix of the radiation impulse responses. The vector of external forces $f(t) \in \mathbb{R}^{m}$ is given by $f(t)=f_{e}(t)+f_{\text {pto }}(t)$ where $f_{e}(t)$ is the exciting force and $f_{\text {pto }}(t)$ is the PTO force. The excitation force is calculated as $f_{e}(t)=\mathscr{F}^{-1}\{\mathrm{X}(\omega) \eta(\omega)\}$, where $\eta(\omega)$ is the Fourier transform of the wave elevation and $X(\omega)$ is the frequency domain exciting force transfer function.

\subsection{Numerical modelling}

The hydrodynamic coefficients $\mathrm{m}_{\infty}$ and $\mathbf{K}(t)$ are calculated from the frequency domain radiation impedance matrix $\mathbf{Z}(\omega)$ by applying Ogilvie's relations [25]. The matrices $\mathbf{X}(\omega), \mathbf{Z}(\omega), \mathbf{M}$ and $\mathbf{S}$ are then computed in the boundary element solver WAMIT () [26]. This package is widely applied in studies of WEC arrays, as in [11,12]. In WAMIT the high order method is used and computations are performed for 160 frequencies equally spaced at intervals of $0.0151 \mathrm{rad} / \mathrm{s}$. All control simulations are done in MATLAB ${ }^{\circledR}$ and are performed with the same frequency resolution as the WAMIT output. The software simulates the motion of the devices, the PTO forces, the instantaneous converted power and the vertical velocities and displacements of the WECs for a representative surface elevation time-history of a given wave spectrum.

\subsection{Viscous Damping Approximation}

The viscous effect of a fluid on a body is usually described, under certain conditions [27], as a force proportional to the square of the relative velocity between the body and the fluid surrounding the body; that is $f_{v}=(1 / 2) A C_{d} \rho \dot{z}|\dot{z}|$, where $\rho$ is the fluid density, $A$ is the area of the body projected onto the plane orthogonal to the velocity $\dot{z}$, and $C_{d}$ is the drag coefficient, which is obtained experimentally (see for example appendix 2 in [27]). Several recent studies have considered the effects of viscosity on WEC motion, such as [20, 28, 29]. The objective of the approximation is to find a force $\hat{f}_{v}$ which is linearly proportional to the velocity and that dissipates the same amount of energy as the force $f_{v}$. This procedure is known as Lorentz linearisation [30], and it has been used in the case of WECs by Folley et al. [31]. The approximation is carried out by equating the work of the nonlinear force $f_{v}$ with the work of the linear approximation $\hat{f}_{v}=B_{v} \dot{z}$ which results in $\int_{0}^{T} f_{v}|\dot{z}| d t=\int_{0}^{T} \hat{f}_{v} \dot{z} d t$. By means of simple manipulations, the damping coefficient $B_{v}$ can be expressed as

$$
B_{v}=\frac{C_{d} \rho A \int_{0}^{T} \dot{z}^{2}(t)|\dot{z}(t)| d t}{2 \int_{0}^{T} \dot{z}^{2}(t) d t}
$$

Knowledge of the velocity in Eq. (4) is required to calculate the coefficient $B_{v}$, but the velocity itself depends on $B_{v}$, therefore an iterative procedure has been implemented. The method is initiated by setting $B_{v}$ to an initial value $B_{v}^{0}$, which is not critical for the convergence because the relation between $B_{v}$ and the velocity $\dot{z}$ is monotonic ( $\dot{z}$ decreases when $B_{v}$ increases). The $i$-th iteration is composed of two steps, which are:

1) Calculate the velocity $\dot{z}$ using the value of $B_{v}^{i-1}$

2) Calculate $B_{v}^{i}$ using the velocity $\dot{z}^{i}$ and the formula 4

The procedure is stopped when the difference between two subsequent values of $B_{v}$ is smaller than a threshold $\delta$, that is $\left|B_{v}^{i}-B_{v}^{i-1}\right| \leq \delta$. For any given geometry, controller type and sea state, the coefficient $B_{v}$ is calculated by simulating an isolated device.

\section{ENERGY MAXIMIZING CONTROL AND SEA-STATE BASED TUNING}

Two control strategies are considered for the control of WEC arrays, named Global Control (GC) and Passive Tuning (PT). GC is based on a centralised control algorithm which uses the complete model of the array whereas PT is a fixed sea-state based tuning scheme. It is assumed that the total hydrodynamic force on each WEC can be estimated for GC, where the total hydrodynamic force is intended to be the sum of the force exerted by the incoming wave, diffraction and radiation. The control problem is defined as: find the optimal profile for the PTO forces which maximise the total energy absorbed by the array described by the equation of motion (3) over a time interval of length $T$ and, eventually, satisfying constraints associated with the motion of WECs. The total energy absorbed by the array is considered to be sum of the mechanical work performed by each of the PTO forces, and it is formulated as

$$
E=-\sum_{k=1}^{n} \int_{0}^{T} \dot{z}^{k}(t) f_{p t o}^{k}(t) d t
$$

where $\dot{z}^{k}(t)$ and $f_{\text {pto }}^{k}(t)$ are, respectively, the heave velocity and the PTO force of the $k$-th device. 


\subsection{Discretisation}

The control problem is discretised by approximating the velocity and the PTO force with a linear combination of basis function, resulting in a finite dimensional optimisation problem. In this paper, trigonometric functions are chosen as basis functions, thus the PTO force and the velocity are approximated with the truncated zero-mean Fourier series

$$
\begin{aligned}
\dot{z}^{k}(t) & \approx \sum_{n=1}^{N / 2} x_{n, c}^{k} \cos \left(n \omega_{0} t\right)+z_{n, s}^{k} \sin \left(n \omega_{0} t\right) \\
f_{p t o}^{k}(t) & \approx \sum_{n=1}^{N / 2} p_{n, c}^{k} \cos \left(n \omega_{0} t\right)+p_{n, s}^{k} \sin \left(n \omega_{0} t\right)
\end{aligned}
$$

The best approximation of the solution of the equation of motion (3) is sought by applying the Galerkin method, the details of which are presented in [32], and the result is the linear system

$$
\mathbf{G X}=\mathbf{P}+\mathrm{E}
$$

where $X, P, \mathrm{E}$ and $\mathrm{G}$ are defined as

$$
X=\left[\begin{array}{l}
X^{1} \\
X^{2}
\end{array}\right] \quad P=\left[\begin{array}{l}
P^{1} \\
P^{2}
\end{array}\right] \quad \mathrm{E}=\left[\begin{array}{l}
\mathrm{E}^{1} \\
\mathrm{E}^{2}
\end{array}\right] \quad \mathrm{G}=\left[\begin{array}{ll}
\mathrm{G}_{11} & \mathrm{G}_{12} \\
\mathrm{G}_{21} & \mathrm{G}_{22}
\end{array}\right]
$$

The vectors $X^{k}$ and $P^{k}$, for $k=1,2$, are the vectors of the Fourier coefficients of the velocity and PTO force of the $k$-th device, and are arranged as

$$
\begin{aligned}
X^{k} & =\left[x_{1, c}^{k}, x_{1, s}^{k}, x_{2, c}^{k}, x_{2, s}^{k}, \ldots, x_{\frac{N}{2}, c}^{k}, x_{\frac{N}{2}, s}^{k}\right]^{T} \text { for } k=1,2 . \\
P^{k} & =\left[p_{1, c}^{k}, p_{1, s}^{k}, p_{2, c}^{k}, p_{2, s}^{k}, \ldots, p_{\frac{N}{2}, c}^{k}, p_{\frac{N}{2}, s}^{k}\right]^{T}
\end{aligned}
$$

The elements of the vectors $E^{k}$ are the Fourier coefficients of the excitation force on the $k$-th device and are arranged in the same manner as the vectors $X^{k}$ and $P^{k}$. The matrices $\mathrm{G}_{i j} \in \mathbb{R}^{N \times N}$ composing the matrix $\mathbf{G}$ are block diagonal, where each of the $N / 2$ blocks is of size two and the $l$-th block is defined as

$$
\begin{aligned}
\mathrm{G}_{i j}^{l} & =\left[\begin{array}{cc}
\mathrm{D}_{i j}^{l} & \mathrm{M}_{i j}^{l} \\
-\mathrm{M}_{i j}^{l} & \mathrm{D}_{i j}^{l}
\end{array}\right] \quad \text { for } l=1, \ldots, N / 2 \\
\mathrm{D}_{i j}^{l} & =\mathrm{R}_{i j}\left(l \omega_{0}\right)+\mathrm{B}_{i j} \\
\mathrm{M}_{i j}^{l} & =l \omega_{0}\left(\mathrm{M}_{i j}+\mathrm{m}_{i j}\left(l \omega_{0}\right)\right)-\mathrm{S}_{i j} /\left(l \omega_{0}\right) .
\end{aligned}
$$

$\mathbf{B}_{i j}, \mathbf{M}_{i j}$ and $\mathrm{S}_{i j}$ are, respectively, the elements of the matrices $\mathbf{B}$, $\mathbf{M}$ and $\mathbf{S}$, while $\mathrm{R}_{i j}(\omega)$ and $\mathrm{m}_{i j}(\omega)$ are the elements of the radiation impedance matrix $Z(\omega)$, which is computed by WAMIT and it is defined as $\mathbf{Z}(\omega)=\mathrm{R}(\omega)+i \omega \mathrm{m}(\omega)$.

\subsection{Global Control}

The control system of the GC strategy is aware of the whole configuration of the array; the resulting optimisation problem is defined by the cost function $\mathrm{W}=-P^{T} X$, which is obtained by substituting (6) and (7) into the definition of the total absorbed energy in [5]. If $\mathrm{G}$ is non-singular, the cost function can be expressed as a function of $P$ by solving (8) w.r.t $X$, and the coefficients $P^{\star}$ of the optimal PTO forces that maximise the absorbed energy for the array are obtained by solving the optimisation problem

$$
P^{\star}=\arg \max _{P}-P^{T} X=-P^{T} \mathrm{G}^{-1} P-P^{T} \mathrm{G}^{-1} \mathrm{E}
$$

Equation 10 is an example of a quadratic program. It can be verified that the matrix $G$ of the quadratic cost function 10 is positive definite. The active set algorithm is used to solve the optimisation problems, which is implemented in Matlab by the function quadprog.

\subsection{Passive Tuning}

While not a energy-maximizing control scheme per se, PT will serve as a useful benchmark against which we can measure performance of GC presented in 5, in addition enabling us to compare our results to previously published data [12, 17]. Following [15] for single devices and [6, 17], $B_{\text {pto }}$ is a chosen a constant for each device set to maximize the power extracted at a given frequency $\omega$. In [15] it was shown that for a single sea state, tuning $B_{\text {pto }}$ to the peak frequency $\omega_{p}$ gives better results than tuning to the energy frequency $\omega_{e}$. However, this does not necessarily have to be the case in a mixed sea state where we tune to the average of many sea states and not a specific one. In light of the fact that $T_{e}$ is lower than $T_{p}$ and is closer to the resonance period of the modelled devices, and that the mode of $T_{p}$ is lower peak period than $T_{p} a v$, we have chosen to tune the devices to $T_{e} a v$. For each modelled Geometry, $T_{e} a v$ excludes those sea states that exceed the exclusion threshold as defined in Sec. 5.2 We recognize that the difference in tuning is likely to be small compared to the difference between the performance of GC and PT.

For each device in the array $B_{\text {pto }}$ is given by the following equation [8, 17, 33]:

$$
\mathrm{B}_{\mathrm{pto}}=\sqrt{\left[\mathrm{B}\left(\omega_{e}\right)\right]^{2}+\omega_{e}^{2}\left[\mathrm{M}+\mathrm{m}\left(\omega_{e}\right)-\frac{\mathrm{S}}{\omega_{e}^{2}}\right]^{2}}
$$

Here $M$ is the generalized mass matrix, $S$ is the hydrodynamic stiffness, $\mathrm{B}\left(\omega_{e}\right)$ and $\mathrm{m}\left(\omega_{\mathrm{e}}\right)$ are the values of the radiation damping and added mass at the tuning frequency $\omega_{e}$. All matrices are 
$\in \mathbb{R}^{m \times m}$. We find the energy by solving the system in Eq. 8 , where

$$
\mathrm{P}=-\mathbf{B X}
$$

is a matrix of the PTO forces. B is the identity matrix $\mathrm{I} \in \mathbb{R}^{m \times m}$ multiplied by $\mathrm{B}_{\text {pto }}$ for each device. Rearranging Eq. (8) gives the Fourier coefficients of velocity

$$
X=\frac{E}{\mathbf{G}+\mathbf{B}}
$$

The total energy absorbed by the array, given in (5), can then be written as

$$
E=\mathbf{B} \mathrm{X}^{\mathrm{T}} \mathrm{X}
$$

where ${ }^{\mathrm{T}}$ indicates vector transpose. This is equivalent to the frequency domain representation as detailed in [2].

\section{Results}

\subsection{Modelled array layouts}

TABLE 2. MODELLED CYLINDER PARAMETERS

\begin{tabular}{|ccccc|}
\hline Geometry & Radius $[\mathrm{m}]$ & Draught $[\mathrm{m}]$ & Resonance Period $T_{0}[\mathrm{~s}]$ & $T_{e} a v / T_{0}$ \\
\hline I & 3.5 & 13 & 7.38 & 1.28 \\
\hline II & 5 & 6 & 5.92 & 1.57 \\
\hline III & 7.25 & 3 & 5.08 & 1.71 \\
\hline
\end{tabular}

Using the wave data presented in Sec. 2 and the two control schemes detailed in Sec. 4, we run a set of simulations for cylinders of three different shapes described for separation distances logarithmically spaced from $2.2 r$ to $40 r$. The three devices have approximately the same volume $\left(\lesssim 16 \pi \mathrm{m}^{3}\right)$ but different resonance periods and radiation properties: with cylinder geometry I (henceforth referred to as Geometry I) being the least radiative and Geometry III the most. Table 2 lists the radii $r$, drafts $h$,resonant periods $T_{0}$, and the ratio of the $T_{e} a v$ used in PT to $T_{0}$ for each device. The parameters $\omega_{0}$ and $N$ for the discretisation of the control problem are $N=160$ and $\omega_{0}=2 \pi / 180$ $\mathrm{rad} / \mathrm{s}$ respectively, while the threshold for the adaptive approximation of the viscous damping 3.3 is $\delta=1$. As was discussed in Chapter 6 of [33], regular polygon array configurations each have areas of constructive and destructive interference that occur at specific incident wave angle which are due to the interplay between the shadowing and radiation effects. For example, as was
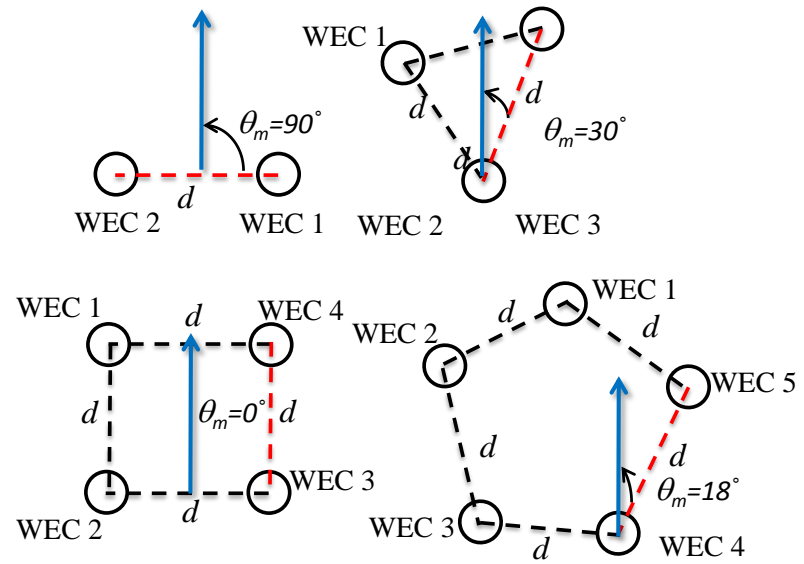

WEC 2

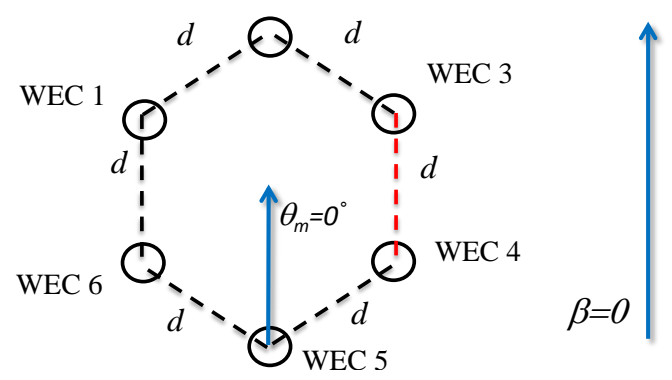

FIGURE 3. TOP VIEW OF 2,3,4,5, AND 6 BODY CIRCULAR ARRAY. ALL SEPARATION DISTANCES $d$ ARE REGULAR POLYGON SIDES. THE ARRAYS ARE ORIENTED SO THAT $\theta_{m}$ IN Eq.(1) IS ALIGNED WITH $\beta=0$ (SHOWN IN BLUE ARROW) AS DEFINED IN THE WAMIT $囚$ COORDINATE SYSTEM. The WAMIT $R X$-AXIS IS SHOWN IN RED. THE DIRECTION OF INCOMING WAVES IS FROM THE BOTTOM

shown in Fig. 6.12 in [33], for a three body arrangement of a GCcontrolled cylinder of Geometry III, there are broad areas of constructive interference when the array when the incident wave is coming at $30^{\circ}$ to the triangular array principle axis. Accordingly, we arrange the arrays as shown in Fig. 3 so that a wave coming from the bottom creates areas of maximum constructive interferences at close distances $(d \lesssim 20 r)$. At our study site, we assume this direction is aligned with the mean direction of the incoming waves, $\theta_{a v}=281.7^{\circ}$. We then model the angular spread around $\theta_{a v}$ by using Eq. (1) to create a weighting function that in effect

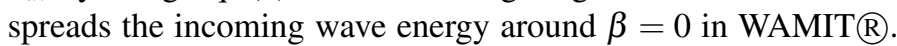
The sum of energies over all the incident angles is then equal to one. Because of the symmetry of the layouts and the directional distribution $\beta$ is modelled from $0^{\circ}$ to $90^{\circ}$ with increments of $5^{\circ}$.

\subsection{Yearly average power output}

The optimal configurations are determined by the highest mean annual production for a specific control scheme and geometry. To produce this number for a WEC in a given wave 
climate is usually obtained by multiplying the power matrix for a given set of pairs $\left(T_{e}, H_{m 0}\right)$ by the power matrix of the device [12,20, 21]. An example power matrix for a PT-tuned single device of Geometry II is shown in Fig. 4. The matrices are created by simulating the WECs with a wave elevation given by the average sea state spectra in each $\left(T_{e}, H_{m 0}\right)$ bin for $T=180 \mathrm{sec}$. Because we are looking for the average response of the device over a long time period, that is a year, and not on the response to a constantly changing sea state, the length of simulation time is sufficient to accurately represent the power capture of each control scheme. Furthermore, $T$ is long enough for the simulation to reach a steady state where the transient response is no longer significant. Given the likelihood that the largest sea states will cause the device to go into survival mode, we set a criteria to exclude sea states with $H_{m 0}$ greater than the draft, similar to the procedure in [21]. This results in a zero power cut-off at $H_{m 0}=$ $6 m$ and $3 m$, respectively for Geometries II and III. Given its draft of $13 m$, device I is able to operate in all significant sea states.

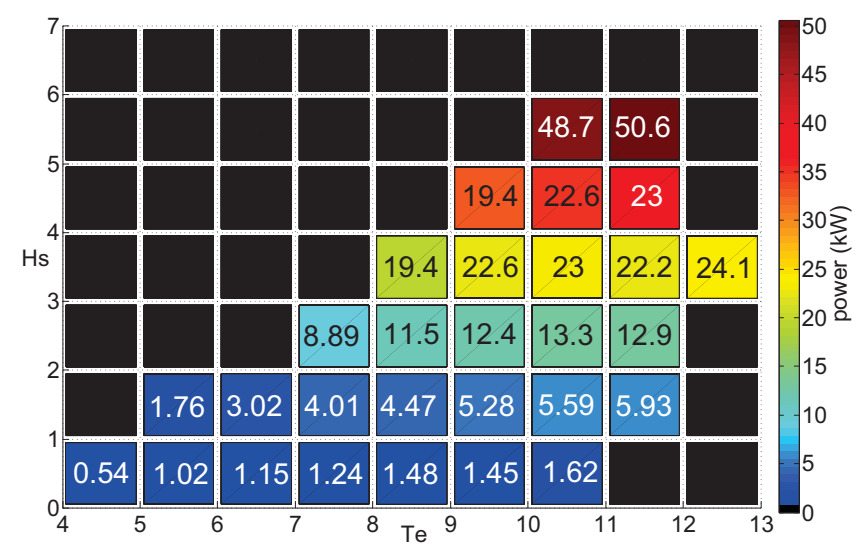

FIGURE 4. POWER MATRIX FOR PT-TUNED SINGLE DEVICE RADIUS 5m DRAUGHT 6m GEOMETRY II

Similar power matrices were produced for both GC and PT for all array configurations for all inter-device spacings $d$ (not shown). The resulting power matrices were multiplied by the probability of occurrence given by the sea state matrix (Fig. 2) to give the annual mean absorbed power in $\mathrm{kW}$. For each control type, device shape, and array size, optimal configurations were chosen that resulted in the highest mean yearly converted power. The results are presented in Sec. 5.4. In Sec. 5.3, we look at the $d$, the independent variable which determines our optimal configurations.

\subsection{Influence of separation distance}

Because of hydrodynamic interactions in the array, power yield is dependent on the separation distance $d$, most notably for
GC, where for short separation distances of less than 5 diameters power production for arrays of multiple bodies falls off up to 25 $\%$ from that of the same number of independent bodies. This difference is due to the greater motions experienced by a device controlled by GC which modify the wave field around it, causing negative interference by radiation. This effect can be seen in figs. 5 and 6 where the normalized power (divided by the power converted by a single device of the same Geometry and control type) for one to 6 devices is shown for $d$ from $2 d$ to $40 d$ for Geometry I, and III for the bin $H_{m 0}=2-3 m T_{e}=8-9 \mathrm{~s}$. (Geometry II is similar to III and not shown) Since this the most frequently encountered sea state at the AMETS site (see Fig. 2), accounting for $7.48 \%$ of all sea states, it will influence markedly the optimal layouts which are presented in Sec. 5.4 .

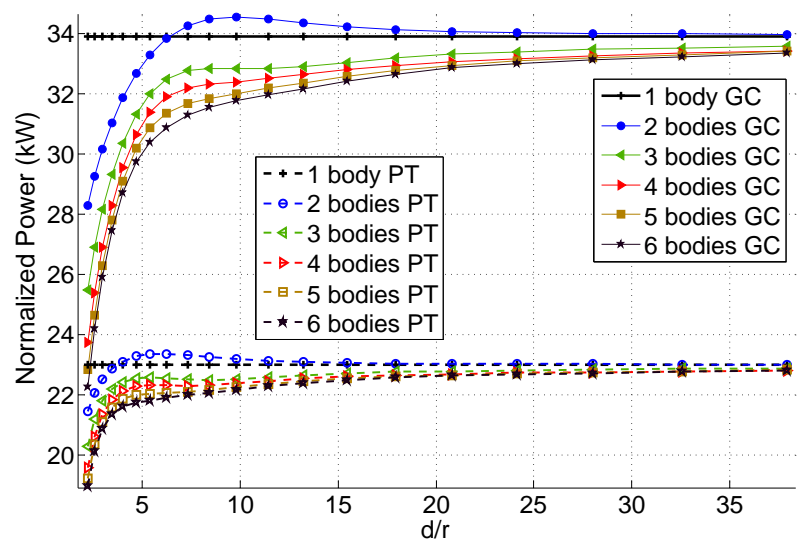

FIGURE 5. AVERAGE POWER CONVERTED VS. SEPARATION DISTANCE. GEOMETRY III. $H s=2-3 m$ Te $=8-9 s$.

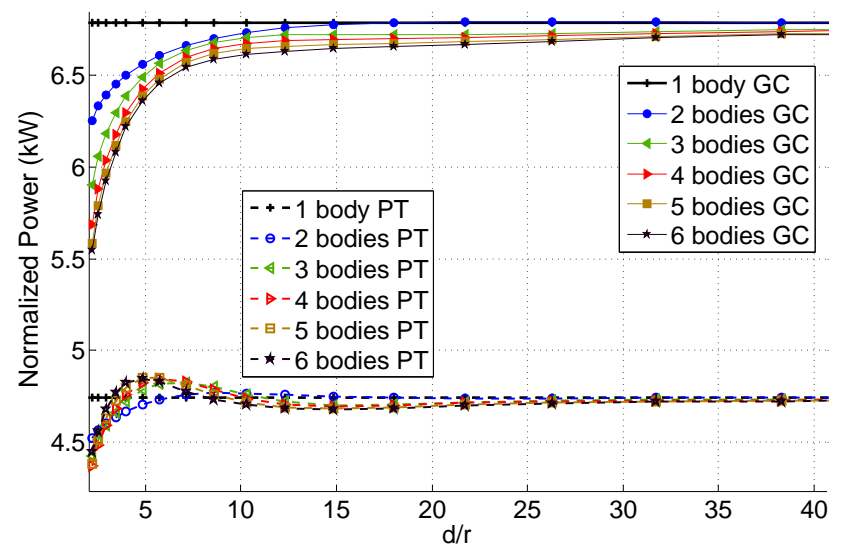

FIGURE 6. AVERAGE POWER CONVERTED VS. SEPARATION DISTANCE. GEOMETRY I. $H_{m 0}=2-3 m T_{e}=8-9 s$. 
We can note that GC outperforms PT for all cases, with the qualifier that for the closest separation distances the difference is half as large as for $d$ of 10 and up. Comparing the curves for GC and PT we see that in each case, the 2-body array is the most advantageous in terms of relative power capture, with wide areas of $d$ for which the interference is constructive. It is illuminating to observe that despite their similar shapes, the peak of the GC curve occurs at a larger $d$ than that for PT, as we will see in detail in Sec. 5.4 Also of note is the performance of PT for Geometry I, for which the multi-body arrays have an area of constructive interference at a $d / r$ of around 5 .

\subsection{Optimal array configurations}
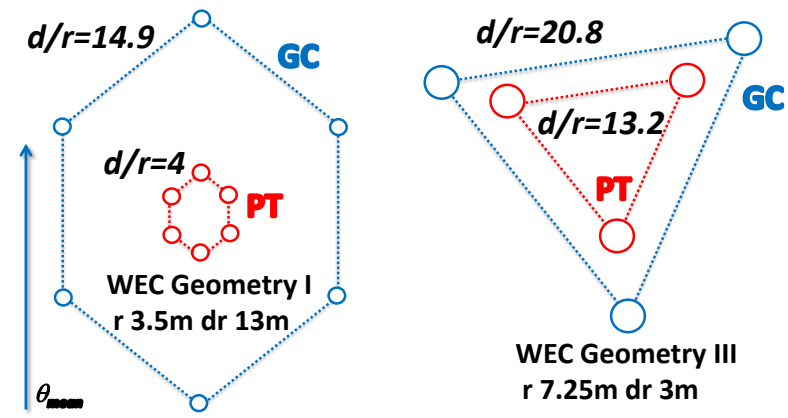

FIGURE 7. OPTIMAL LAYOUT FOR THREE AND SIX BODY ARRAY. GEOMETRY I. PT (red) AND GC (blue) (TO SCALE)

In Sec. 5.3 we saw that for a given device geometry, control, and array size, $d$ plays a crucial role in determining the total power output for a given sea state. Out of all the power matrices created for each modelled scenario, as described in sec 5.2 we select those configurations that give the highest mean annual power for each control scheme. An example is shown in Fig. 7 for Geometries I and III for 3 and 6 bodies. Except for the 2body case, this is the largest possible $d$ beyond which there are negligible gains for further separation, as can be evidenced in the asymptotic behaviour of the curves in figs. 5 and 6 . However, since our aim is to find the optimal closely-spaced configurations, we can slightly relax the optimality condition and look at the cases with a power capture $1 \%$ less than the optimum. The results, given as the annual mean power capture normalized by the power extracted by one PT-controlled body and by the number of bodies are displayed in Tab. 3, with the single device power capture also shown for reference.

The results demonstrate that the small trade-off in power capture decreases the necessary separation distance substantially from its maximum of $40 \mathrm{~d}$. Even so, there are significant differences between the control schemes. We can observe that the suboptimal $d / r$ for GC is on average twice greater than that for
PT, indicating the need for increased inter-device spacing for GC to take full advantage of constructive interference. This effect is magnified by the radiation properties of the cylinders, with the most radiative Geometry III requiring greater $d / r$ for best performance compared to the least radiative Geometry I. The decrease in the resonance period from Geometry I to Geometry III also modifies the cylinder response, however, the effect is not as significant as that of wave radiation. Moreover, GC is able to modify the apparent $T_{0}$ by varying the damping to match the frequency of the incoming waves. In Sec. 5.3 we saw that for a given device geometry, control, and array size, $d$ plays a crucial role in determining the total power output for a given sea state. Out of all the power matrices created for each modelled scenario, as described in sec 5.2. we select those configurations that give the highest mean annual power for each control scheme. An example is shown in Fig 7 for Geometries I and III for 3 and 6 bodies. Except for the 2-body case, this is the largest possible separation distance beyond which there are negligible gains for separating further, as can be evidenced in the asymptotic behaviour of the curves in figs. 5 and 6 .

TABLE 3. ANNUAL MEAN POWER NORMALIZED AGAINST PT FOR ONE DEVICE FOR SUBOPTIMAL CONFIGURATIONS FOR GC AND PT AT THE AMETS SITE.

\begin{tabular}{|c|c|c|c|c|c|c|c|}
\hline \multirow{3}{*}{$\mathrm{N}$} & & \multicolumn{3}{|c|}{ PT } & \multicolumn{3}{|c|}{ GC } \\
\hline & & Geo I & Geo II & Geo III & Geo I & Geo II & Geo III \\
\hline & $P(k W)$ & 4.27 & 10.2 & 8.54 & 7.02 & 16.5 & 14.2 \\
\hline \multirow[b]{2}{*}{2} & $P / P_{1 P T}$ & 0.99 & 0.99 & 1 & 1.63 & 1.61 & 1.7 \\
\hline & $d / r$ & 5.7 & 4.8 & 4.69 & 12.3 & 11 & 8.41 \\
\hline \multirow[b]{2}{*}{3} & $P / P_{1 P T}$ & 1 & 0.987 & 1.17 & 1.62 & 1.59 & 1.6 \\
\hline & $d / r$ & 5.71 & 4 & 13.2 & 12.3 & 13 & 20.8 \\
\hline \multirow[b]{2}{*}{4} & $P / P_{1 P T}$ & 1 & 0.985 & 1.17 & 1.62 & 1.59 & 1.6 \\
\hline & $d / r$ & 4.86 & 4.8 & 15.4 & 12.3 & 15.6 & 24.1 \\
\hline \multirow[b]{2}{*}{5} & $P / P_{1 P T}$ & 1 & 0.985 & 1.17 & 1.61 & 1.58 & 1.6 \\
\hline & $d / r$ & 4.86 & 5.6 & 17.9 & 14.9 & 21.8 & 28 \\
\hline \multirow{2}{*}{6} & $P / P_{1 P T}$ & 1 & 0.984 & 1.17 & 1.61 & 1.58 & 1.6 \\
\hline & $d / r$ & 4 & 15.6 & 17.9 & 14.9 & 25.8 & 28 \\
\hline
\end{tabular}

Geometries (rad. (m), dr. (m)) I: $(3.5,13)$ II: $(5,6)$ III: $(7.25,3)$

We note is that in this wave climate, the majority of cases demonstrate diminished power uptake compared to the isolated case, with the exception of Geometry III for the 2-body case. Nonetheless constructive interference for Geometry III does not extend to multi-body configurations: all of them have negative interference at close $d$ and as a consequence the suboptimal dis- 
tances are quite far out compared to the other devices. This indicates that the benefits of choosing a highly radiative WEC will not be fully realized if many devices are placed together, and it may be more economically expedient to choose an alternate geometry. Yet we can also confirm that the array interactions, though negative, are not severely detrimental to total power capture, even for the multi-body arrays, and that devices can be placed close enough together with minimal loss in performance, while still economizing on cables and other array infrastructure.

\section{DISCUSSION}

As witnessed in Sec. 5, for a given set of fixed variables with a goal of power maximization, the control scheme plays the biggest role in determining the optimal configuration of an array. There is a clear trade-off in between the increased power output of a GC-controlled array and the required $d$ for its optimal performance. In the modelled cases in this paper, GC outperforms PT at all separation distances. However, the increased costs associated with implementing a reactive control scheme with the infrastructure necessary for inter-device communication can outweigh the economic benefit from the improved power capture. Moreover, the knowledge that increasing the separation distance for the multi-body configurations from $2.2 r$ to $10 r$ increases the power capture by a more than a third for the GC-modelled scenarios (refer to figs. 5 and 6), is useful for developers weighting the costs of infrastructure vs. the improved array performance.

In contrast to previous studies that have looked at array configurations such as [5, 6, 12], we have utilized real spectral data from a potential WEC array site. Furthermore, the proposed optimal control methods can be applied in real-time, unlike that in [13]. The frequency and directional spread of the data leads to a smoothing effect on the power output of the array: it is more difficult to get constructive interference. Although not explicitly studied in this paper, the fact that the power output of a circular array in a spectral wave climate produce is robust to changes in $d$ is useful from several viewpoints. Firstly, because of the possibility that loosely moored devices can deviate from their set positions, the power capture of a specific configuration can in fact degrade as the devices are displaced from the optimal positions that were designed to maximize power capture [14]. Secondly, because of power requirements for the grid, power smoothing can be a more important consideration in array layout design than maximal power capture, as seen in [11, 18].

\section{CONCLUSIONS}

In this paper we have seen that the control scheme plays a key role in determining the optimal separation distance in a WEC array operating in real seas, with the reactive GC scheme requiring greater separations distances for optimal performance than the passive PT scheme. While this study is limited in it its mod- elling scenario, it does underscore the role that a wave climate plays in a determining the optimal array layout for a given control scheme: in the case of AMETS site the sea states that contribute the most to the power production force greater separation distances for optimal performance of GC vs. PT.

Compared to previous optimization studies that have shown significant improvements in array power capture for certain configurations in regular waves such as [3, 4, 6], we have shown that for a real wave climate the opportunity to increase the array power capture over that of isolated devices is small. However, we have also seen that in such a wave climate the benefit of a control scheme that can calculate optimal motions in real time is significant regardless of number of devices, with a increase in the mean annual power yield of GC around 1.5 times that of PT for Geometries I and II to nearly 2-fold increase for the power yield for Geometry III.

Furthermore, the power output for a controlled circular array is relatively insensitive for a large range of $d$, allowing developers the freedom in placing WECs with the knowledge that array performance would not severely degrade should the separation distance be different from the originally planned. Given the uneven bathymetry and issues that may arise during installation such a scenario is quite likely at the AMETS site and other array sites under consideration. The extremely energetic sea states encountered at Belmullet suggest a power maximization control scheme might only be useful for smaller sea states. In this case, a scheme like GC will have an advantage over a sea-state based tuning scheme in that it allows for a device with a large draft designed to withstand great oscillations to be tuned to a smaller sea state with a smaller peak period. One possibility of implementing a hybrid control scheme would be to use motion constraints, such as those implemented for GC in [19].

A number of factors were not considered in the study that would need to be addressed in future work. In addition to considering motion constraints, it would be useful to look at devices oscillating in more than one mode of motion. Moreover, a more realistic geometric model of the devices would give a more precise number for the annual power capture. Finally, it is important to remember that any WEC array hydrodynamical layout optimization and control algorithms have to be included in a much broader wave to wire model that includes all other economic and technical parameters which ultimately will determine the success of a wave energy project.

\section{ACKNOWLEDGMENT}

The authors gratefully acknowledge the financial support of Enterprise Ireland under contract TD/2009/0331 and Sustainable Energy Authority of Ireland for providing the wave data. The first author would like to thank Dr. Evgenia Balitskaia for her help and critique in analysing the sea state model. 


\section{REFERENCES}

[1] Evans, D., 1979. "Some theoretical aspects of three-dimensional wave-energy absorbers". In Sympoisum on Ocean Wave Energy Utliziation.

[2] Falnes, J., 1980. "Radiation impedance matrix and ship optimum power absorbtion for interacting oscillators in surface waves". Applied Ocean Research, 2, pp. 75-80.

[3] Thomas, G. P., and Evans, D., 1981. "Arrays of three-dimensional wave-energy absorbers". Journal of Fluid Mechanics, 108, pp. 67-88.

[4] McIver, P., Mavrakos, S., and Singh, G., 1996. "Wave-power absorption by arrays of devices". In Proceedings of the 2nd European Wave Power Conference, Lisbon, Portugal pp.126-133.

[5] Fitzgerald, C., and Thomas, G. P., 2007. "A preliminary study of the optimal formation of an array of wave power devices". In Proceedings of the 7th European Wave and Tidal Energy Conference, Porto, Portugal.

[6] Child, B., and Venugopal, V., 2010. "Optimal configurations of wave energy devices". Ocean Engineering, 37, pp. 1402-1417.

[7] Ricci, P., Saulnier, J.-B., and de O. Falcão, A. F., 2007. "Pointabsorber arrays: a configuration study off the portuguese west coast". In Proceedings of the 7th European Wave and Tidal Energy Conference, Porto, Portugal.

[8] Bellew, S., T., S., and Stansby, P., 2009. "Optimisation of a heterogeneous array of heaving bodies (ewtec)". In Proceedings of the 8th European Wave and Title Energy Conference, Uppsala, Sweden. pp. 519-527.

[9] Borgarino, B., Babarit, A., and Ferrant, P., 2011. "Impact of the separating distance between interacting wave energy converters on the overall energy extraction of an array". In Proceedings of the 9th European Wave and Tidal Energy Conference, Southampton, UK.

[10] Borgarino, B., Babarit, A., and Ferrant, P., 2012. "Impact of wave interaction effects on energy absorbtion in large arrays of wave energy converters". Ocean Engineering, 41, pp. 79-88.

[11] Cruz, J., Mackay, E., Livingstone, M., and Child, D., 2013. "Validation of design and planning tools for wave energy converters (wecs)". In Proceedings of the 1st Marine Technology Symposium METS13 Washington, D.C.

[12] Vicente, M., and Sarmento, A., 2013. "Layout optimization of wave energy point absorber arrays". In 10th European Wave and Tidal Energy Conference Series, Aalborg, Denmark.

[13] Folley, M., and Whittaker, T., 2009. "The effect of sub-optimal control and the spectral wave climate on the performance of wave energy converter arrays". Applied Ocean Research, 31(4), pp. 260-266.

[14] Bacelli, G., Balitsky, P., and Ringwood, J. V., 2013. "Coordinated control of arrays of wave energy devices - benefits over independent control". IEEE Transactions on Sustainable Energy, 4, pp. 1091 - 1099.

[15] Yavuz, H., Stallard, T. J., McCabe, A. P., and Aggidis, G. A., 2006. "Time series analysis-based adaptive tuning techniques for a heaving wave energy converter in irregular seas". Proceedings of the Institution of Mechanical Engineers, Part A: Journal of Power 1 Energy, 221, pp. 77-90.

[16] Justino, P. A., and Clément, A., 2003. "Hydrodynamic perfor- mance for small arrays of submerged spheres". In Proceedings of 5th European wave energy conference. Cork, Ireland. pp.266-273, pp. 266-273.

[17] Child, B., and Venugopal, V., 2008. "Non-optimal tuning of wave energy device arrays". In 2nd International Conference on Ocean Energy (ICOE),Brest, France.

[18] Engstrom, J., Eriksson, M., Goteman, M., Isberg, J., and Leijon, M., 2013. "Performance of large arrays of point absorbing direct-driven wave energy converters". Journal of Applied Physics, 114(20).

[19] Bacelli, G., and Ringwood, J., 2013. "Constrained control of arrays of wave energy devices". International Journal of Marine Energy, 3-4, pp. 53-69.

[20] Babarit, A., Hals, J., Muliawan, M., Kurniawan, A., Moan, T., and Krokstad, J., 2012. "Numerical benchmarking study of a selection of wave energy converters". Renewable Energy, 41, pp. 44-63.

[21] de Andrés A.D., R.Guanche, Armesto, J., F.delJesus, C.Vidal, and I.J.Losada, 2013. "Time domain model for a two-body heave converter: Model and applications". Ocean Engineering, 72, pp. 116123.

[22] Cahill, B., and Lewis, T., 2013. "Wave energy resource characterisation of the atlantic marine energy test site". International Journal of Marine Energy, 1, pp. 3-15.

[23] Kuik, A., van Vledder, G., and Holthuijsen, L., 1988. "A method for the routine analysis of pitch-and-roll buoy wave data". Journal of Physical Oceanography, 18, pp. 1024-1034.

[24] Cummins, W., 1962. "The impulse response function and ship motions". Schiffstechnik, 9, pp. 101-109.

[25] Ogilvie, T., 1964. "Recent progress toward the understling and prediction of ship motions". In Proc. Fifth Symposium on Naval Hydrodynamics, Bergen, Norway.

[26] WAMIT Inc, 2006. User Manual, Versions 6.4, 6.4 PC, 6.3, 6.3S$P C$.

[27] Douglas, J. F., Gasiorek, J., and Swaffield, J., 2005. Fluid Mechanics. Pearson/Prentice Hall.

[28] Antonutti, R., and Hearn, G. E., 2011. "Optimisation of pointabsorber arrays". In Proceedings of the 9th European Wave and Tidal Energy Conference, Southampton, UK.

[29] Bhinder, M. A., Babarit, A., Gentaz, L., and Ferrant, P., 2011. "Assessment of viscous damping via 3d-cfd modelling of a floating wave energy device". In Proceedings of the 9th European Wave and Tidal Energy Conference, Southampton, UK.

[30] Terra, G. M., van de Berg, W. J., and Maas, L. R. M., 1995. "Experimental verification of lorentz linearization procedure for quadratic friction". Fluid Dynamics Research, 36, pp. 175-188.

[31] Folley, M., Whittaker, T., and van't Hoff, J., 2007. "The design of small seabed-mounted bottom-hinged wave energy converters". In Proceedings of the 7th European Wave and Tidal Energy Conference, Porto, Portugal.

[32] Bacelli, G., Ringwood, J. V., and Gilloteaux, J.-C., 2011. "A control system for a self-reacting point absorber wave energy converter subject to constraints". In IFAC World Congress, Milan, Italy., pp. 11387-11392.

[33] Balitsky, P., 2013. "Modelling controlled arrays of wave energy converters". Master's thesis, National University of Ireland Maynooth http://eprints.nuim.ie/4487/ 